\title{
Functional Studies of the Bacterial Avirulence Protein AvrPto by Mutational Analysis
}

\author{
Jeff H. Chang, ${ }^{1}$ Christian M. Tobias, ${ }^{3}$ Brian J. Staskawicz, ${ }^{1,2}$ and Richard W. Michelmore ${ }^{1,3}$ \\ ${ }^{1}$ NSF Center for Engineering Plants for Resistance Against Pathogens, University of California-Davis, One \\ Shields Avenue, Davis 95616, U.S.A.; ${ }^{2}$ Department of Plant and Microbial Biology, University of \\ California, Berkeley 94720, U.S.A.; ${ }^{3}$ Department of Vegetable Crops, University of California-Davis \\ 95616, U.S.A. \\ Accepted 4 December 2000.
}

\begin{abstract}
Pseudomonas syringae pathovars expressing avrPto are avirulent on plants expressing the resistance gene Pto. Over 85 mutants of avrPto were generated with multiple strategies, and several assays were used to characterize AvrPto function. Only a core of 95 amino acids of the 164 residues was sufficient for binding Pto in the yeast two-hybrid system. Only nine of 65 mutant proteins of AvrPto with amino acid substitutions, created in planta and in vitro, did not interact with Pto in the Gal4 yeast two-hybrid system, suggesting that AvrPto can tolerate many nonconservative substitutions and still interact with Pto. These nine and 12 additional substitution mutants of AvrPto were characterized further. The ability to elicit a hypersensitive response and the effect on pathogenesis in planta for these 21 mutants of AvrPto were strongly correlated with recognition by Pto in the yeast two-hybrid system. Analyses of two proteins with substitutions H54P or D52G/L65P indicated that these residues may be required for delivery into the host cell and protein stability in the bacterial cytoplasm, respectively. The mutants that no longer interacted with Pto and had modified activities in planta were predicted to have changes in their secondary structure.
\end{abstract}

Avirulence genes of phytopathogenic bacteria encode factors that allow detection of the pathogen by plants expressing the appropriate resistance genes. When a corresponding pair of genes are expressed, a resistance response is elicited. This includes the hypersensitive response (HR), an oxidative burst, cross linking of the cell wall, and induction of pathogenesisrelated (PR) genes. Pathogen growth is limited, and disease symptoms are suppressed (Dangl et al. 1996). In the absence of one or both corresponding genes, the pathogen grows to high levels and disease ensues.

Corresponding author: R. W. Michelmore;

Telephone: +1-916-752-1729; Fax: +1-916-752-9659;

E-mail: rwmichelmore@ucdavis.edu

Current address of J. H. Chang: Department of Biology CB \#3280, 108 Coker Hall, Chapel Hill, NC 27599, U.S.A.;

E-mail: jzchang@ucdavis.edu

Current address of C. M. Tobias: DNA Plant Technology Corp., 6701

San Pablo Avenue, Oakland, CA 94608, U.S.A.;

E-mail: tobias@dnap.com
Several observations indicate that avirulence gene products of bacterial pathogens may be intracellular ligands for receptors of disease resistance signaling pathways (Gopalan et al. 1996; Herbers et al. 1992; Leister et al. 1996; Scofield et al. 1996; Tang et al. 1996; Van den Ackerveken et al. 1996). Additionally, a direct molecular interaction between the avirulence protein AvrPto of Pseudomonas syringae pv. tomato and the tomato resistance protein Pto has been demonstrated with the yeast two-hybrid system (Scofield et al. 1996; Tang et al. 1996).

The functions of most avirulence proteins remain unclear. The predicted amino acid sequences of some avirulence proteins have homologies to previously characterized proteins (Hardt and Galan 1997; Swords et al. 1996). Others such as AvrBs3, PthA, and Avrb6 have domains with homology to nuclear localization sequences (NLS). Reporter proteins fused to these NLSs localized to the cell nucleus, suggesting that nuclear localization may be necessary for the function of these avirulence proteins (Van den Ackerveken et al. 1996; Yang and Gabriel 1995). Only one avirulence gene has been shown to encode a product with enzymatic activity. AvrD of $P$. syringae is involved in the production of syringolide, the elicitor recognized by Rpg4 of soybean (Keen et al. 1990).

The hrp genes encode a type III secretion system necessary for avirulence and pathogenicity of phytopathogenic bacteria (Lindgren 1997). Strains of P. syringae with mutations in hrp genes were unable to elicit a HR in resistant plants or cause disease in susceptible plants (Lindgren 1997). Escherichia coli carrying a hrp cluster from Erwinia chrysanthemi secreted avirulence proteins fused to peptide tags, and E. coli carrying a hrp cluster from $P$. syringae elicited a HR when expressing an avirulence gene recognized by the recipient plant (Gopalan et al. 1996; Ham et al. 1998; Mudgett and Staskawicz 1999). Also, many avirulence genes are coordinately induced with hrp genes and have "hrp boxes" upstream of their start codons (Innes et al. 1993; Salmeron and Staskawicz 1993). The type III secretion system also is used by many mammalian bacterial pathogens to secrete and translocate virulence factors into host cells (Hueck 1998).

The avirulence gene avrPto was cloned from $P$. syringae pv. tomato race 0 and encodes a 164 amino acid, hydrophilic protein of $18 \mathrm{kDa}$ with no homologies to known sequences (Ronald et al. 1992). Its expression is dependent upon hrp genes (Salmeron and Staskawicz 1993). The corresponding 
resistance gene Pto has been cloned from tomato and encodes a serine-threonine kinase (Martin et al. 1993). Recognition of AvrPto by Pto has been demonstrated with several different systems. P. syringae pv. tomato and pv. tabaci expressing avrPto elicit a resistance response in tomato and Nicotiana benthamiana, respectively, expressing the resistance gene Pto (Rommens et al. 1995). N. benthamiana plants expressing Pto also develop a HR in response to AvrPto expressed via Agrobacterium tumefaciens (Scofield et al. 1996). In addition, tomato and $N$. benthamiana plants expressing Pto elicit a resistance response to recombinant potato virus $\mathrm{X}$ carrying avrPto (PVX::avrPto) (Tobias et al. 1999). Finally, Pto specifically binds AvrPto in the Gal4 and LexA yeast two-hybrid systems (Scofield et al. 1996; Tang et al. 1996).

This interaction provides the basis for the characterization of the interaction between a resistance protein and its corresponding avirulence protein, and several approaches have been used with the greater emphasis on Pto. The interactions in the yeast two-hybrid system between AvrPto and Pto with amino acid substitutions or as chimeras with Fen parallel the ability of these Pto mutants to elicit a HR in planta (Frederick et al. 1998; Scofield et al. 1996; Tang et al. 1996). In another mutational study of Pto, yeast two-hybrid analysis and $A$. tumefacians-mediated transient expression in planta demonstrated that some forms of Pto were capable of eliciting a HR but did not interact with AvrPto and vice versa (Rathjen et al. 1999). Prior to the current study, complementary mutational studies with AvrPto were limited. Deletion mutants of AvrPto showed corresponding interactions with Pto in the yeast twohybrid system and elicitation of a HR in $N$. benthamiana or Nicotiana tabacum expressing Pto (Scofield et al. 1996; Tang et al. 1996). Recently, nine mutants of avrPto with single amino acid mutations that abolish interactions with Pto in the yeast two-hybrid system were tested for virulence and avirulence activity in planta (Shan et al. 2000).

In this paper, a variety of mutagenesis strategies were used to localize the regions of AvrPto required for Pto recognition, to elicit the HR in planta, and affect pathogenicity of $P$. syringae pv. tabaci. Three separate mutational approaches were used: i) a polymerase chain reaction (PCR)-based strategy to create deletions of increasing size from the amino- and carboxy-termini of AvrPto; ii) infecting plants expressing and lacking Pto with PVX::avrPto to screen for mutants of avrPto generated in planta; and iii) mutagenic PCR to introduce random point mutations into avrPto. Yeast two-hybrid analysis, transient $A$. tumefacians-mediated expression in planta, and pathogenicity assays of $P$. syringae were used to characterize the different aspects of AvrPto function. These studies identified a core of amino acids that were required for function but revealed that AvrPto could tolerate substitutions at many positions and still remain functional.

\section{RESULTS}

\section{Deletion analysis of avrPto.}

A PCR-based strategy was used to introduce incremental deletions of 10 amino acids from amino- and carboxy-termini of AvrPto, either independently or in combination with one another. A total of 20 deletion mutants $(0,20,30,40$, and 60 residues from the amino terminus and 0 or $30,40,50$ from the carboxy terminus and their combinations) were analyzed for interactions with Pto in the Gal4 yeast two-hybrid system. As shown previously (Scofield et al. 1996), 30 amino acids from the carboxy-terminus of AvrPto were dispensable for recognition by Pto. In addition, AvrPto with deletions of 40 amino acids from the carboxy-terminus interacted with Pto. Fragments with 20 and 30 amino acids deleted from the amino-terminus also interacted with Pto (Fig. 1A). In combination, deletions of 30 and 40 amino acids from the amino- and carboxy-termini, respectively, still interacted with Pto. Larger deletions, fragments with 40 and/or 50 amino acids deleted from the amino- and carboxyl-termini, respectively, failed to interact with Pto. In instances where no interactions were detected, Western analysis was used to confirm the expression of Pto and deletion mutant proteins of AvrPto (Chang 1999). No interactions with an empty Gal4 DNA binding domain vector were detected for any deletion mutant of AvrPto. Therefore, a maximum of 95 amino acids in the central region of AvrPto is sufficient for interacting with Pto in the yeast two-hybrid system.

\section{In vivo selection for mutants of avrPto.}

We demonstrated previously that avrPto conferred avirulence on a recombinant virus (Tobias et al. 1999). Wild-type PVX elicited mosaic symptoms in tomato and tobacco, independent of a functional Pto-signaling pathway. PVX::avrPto, however, elicited a rapid resistance response in either tomato cv. Rio Grande $76 \mathrm{R}$ or $N$. benthamiana expressing CaMV35S::Pto, as evidenced by the lack of PVX symptoms and reduction in PVX replication (Tobias et al. 1999). On tomato plants lacking functional Pto or Prf genes or wild-type $N$. benthamiana, PVX::avrPto normally caused varying degrees of necrosis. However, 5 to $10 \%$ of infected plants, regardless of genotype, exhibited wild-type PVX mosaic symptoms, indicating a loss in AvrPto activity. This provided a strategy for selecting mutations in avrPto in planta.

Plants expressing or lacking Pto were infected with PVX::avrPto. RNA was isolated from 24 plants showing potentially wild-type symptoms of PVX infection. The avrPto transcripts were cloned and sequenced to determine the basis for the altered phenotype. This yielded over 15 mutant forms of AvrPto. Multiple mutants were isolated from some plants. The majority of the mutations were small deletions or insertions that resulted in frameshift mutations (Tobias et al. 1999). Five mutant alleles of avrPto carried missense mutations.

Three of these five mutant alleles, avrPto ${ }_{\mathrm{H} 54 \mathrm{P}}$, avrPto ${ }_{\mathrm{A} 62 \mathrm{~S}}$, and $a v r P t o_{\mathrm{W} 116 \mathrm{~T}}$, were identified from two tomato plants lacking functional Pto genes, Rio Grande 76S and pto-18, and two alleles, $a v r P t o_{\mathrm{L} 43 \mathrm{P}}$ and $a v r$ Pto $_{\mathrm{Q} 86 \mathrm{P}}$, were identified from Rio Grande $76 \mathrm{R}$ and $N$. benthamiana expressing CaMV35S::Pto, respectively (Chang 1999). These five mutant alleles of avrPto were individually cloned into pACT2, and their products were tested for interactions with Pto in the Gal4 yeast two-hybrid system. The three mutants identified from plants without functional Pto genes still interacted with Pto, whereas the two originating from Pto-expressing plants did not interact (Fig. 1B). This confirmed that recognition and resistance to the recombinant PVX by Pto is dependent on AvrPto.

\section{PCR-induced mutagenesis of avrPto.}

Mutagenic PCR (Fromant et al. 1995; Leung et al. 1989) was used to generate missense mutations into avrPto. We used 
conditions that introduced approximately one missense mutation at random throughout the $0.5-\mathrm{kb}$ open reading frame (ORF) (Chang 1999). Amplification products were cloned directly into the pACT2 yeast two-hybrid vector, and 114 insert-containing clones were isolated and sequenced. Approximately $38 \%$ of the clones (43/114) were wild type, 5\% had four- or five-point mutations, and $57 \%(65 / 114)$ had one- to three-point mutations per clone. No sequenced clones had insertions, deletions, or nonsense mutations.

Thirty-four clones with one mutation, 20 with two, and six with three mutations were selected for yeast two-hybrid analysis. A total of 72 different residues were altered in these 60 clones. Mutations were distributed evenly throughout the protein and resulted mostly in nonconservative changes (Fig. 2). Surprisingly, the majority of the mutant proteins of AvrPto, $79.4 \%$ (27/34), still interacted with Pto in the yeast two-hybrid system. The total number of unique, altered residues in these 27 proteins accounted for $83.3 \%$ (60/72) of all the amino acid substitutions introduced by mutagenic PCR. Eight mutant proteins of AvrPto with substitutions, D19G, S25P, H41L, P50S, M100I, D115G, D115G/A126T, and P135S (Fig. 1C), were tested for possible interactions with an empty Gal4 DNA-binding domain vector. No $\beta$-galactosidase activity was detected, thus interactions with Pto were specific. One mutant, AvrPto Y89C, showed a weaker interaction with Pto based on the level of $\beta$-galactosidase activity. Seven mutants of AvrPto, S27G/G99V, Q86G/N145S, D52G/L65P, Q86R, G8V/Q42P,

A avPlo NA30 CA40 NA3OXCA40 NA40 CA5O NA40XCA5O

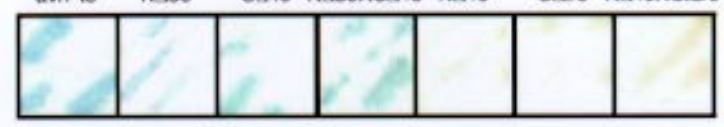

B

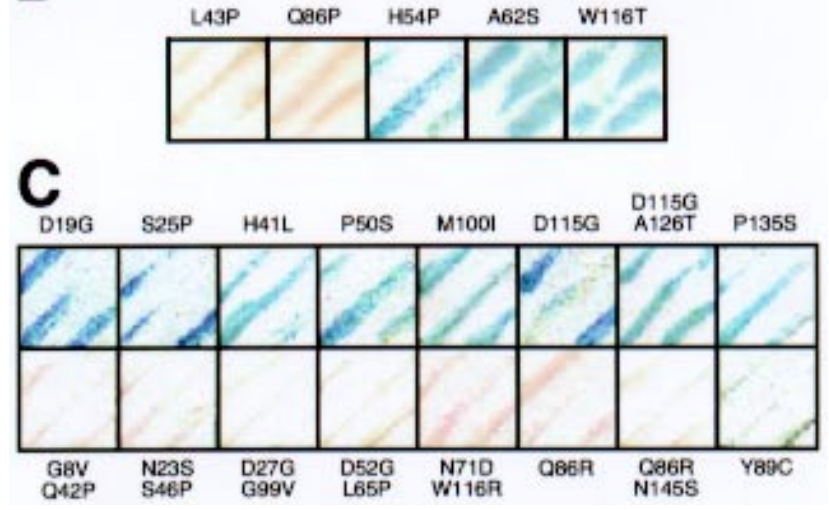

Fig. 1. Gal4 yeast two-hybrid assays for interactions between mutants of AvrPto and Pto. Each panel shows streaks of three independent colonies from each transformation. Blue cells are indicative of a positive interaction. Amino acids are designated as single letters; numbers represent the position of the substitutions. A, Assays for interactions between Pto and AvrPto or deletion mutants of AvrPto. B, Assays for interactions between Pto and substitution mutants of AvrPto identified from PVX::avrPto-infected plants exhibiting wild-type potato virus X (PVX) symptoms. C, Assays for interactions between Pto and substitution mutants of AvrPto generated with mutagenic polymerase chain reaction. Top row: positive interactions with Pto; bottom row: weak or lack of interactions with Pto. Photographs were taken less than $24 \mathrm{~h}$ after incubating cells in X-Gal. These results are representative of at least three separate assays.
N71D/W116R, and N23S/S46P, did not interact with Pto in the yeast two-hybrid system (Fig. 1C). These AvrPto mutants were expressed in yeast cells as detected by Western analysis (Chang 1999).

\section{A. tumefaciens-mediated transient expression of mutant alleles of avrPto in planta.}

To assess their ability to elicit HR, the mutant alleles of avrPto were expressed in planta with A. tumefaciens-mediated transient expression in $N$. benthamiana, with or without CaMV35S::Pto. The five mutant alleles of avrPto identified from PVX::avrPto-infected plants and the 16 PCR-derived mutant alleles described above (Fig. 1B and C) were cloned as transcriptional fusions to the CaMV35S promoter and transformed independently into A. tumefaciens strain A281.

Wild-type avrPto and avrPto mutants did not elicit a phenotype in wild-type $N$. benthamiana following infiltration of A. tumefaciens (Fig. 3A and Table 1). Wild-type avrPto and all 12 avrPto alleles encoding products that interacted with Pto in the two-hybrid system elicited a clear HR in $N$. benthamiana expressing CaMV35S::Pto. The timing and degree of the HR elicited nearly by all was similar to that elicited by A. tumefaciens carrying wild-type avrPto, and tissue collapse was evident approximately $24 \mathrm{~h}$ postinoculation (hpi). N. benthamiana expressing CaMV35S::Pto, however, developed a weaker, delayed HR in response to transient expression of avrPto $_{\mathrm{Y} 89 \mathrm{C}}$ relative to wild-type avrPto. Tissue collapse was not visible until approximately 48 hpi. In contrast, the nine avrPto alleles encoding products that did not interact with Pto in the two-hybrid system also did not elicit a HR in N. benthamiana expressing CaMV35S::Pto. Therefore, there was a tight correlation between binding of AvrPto and

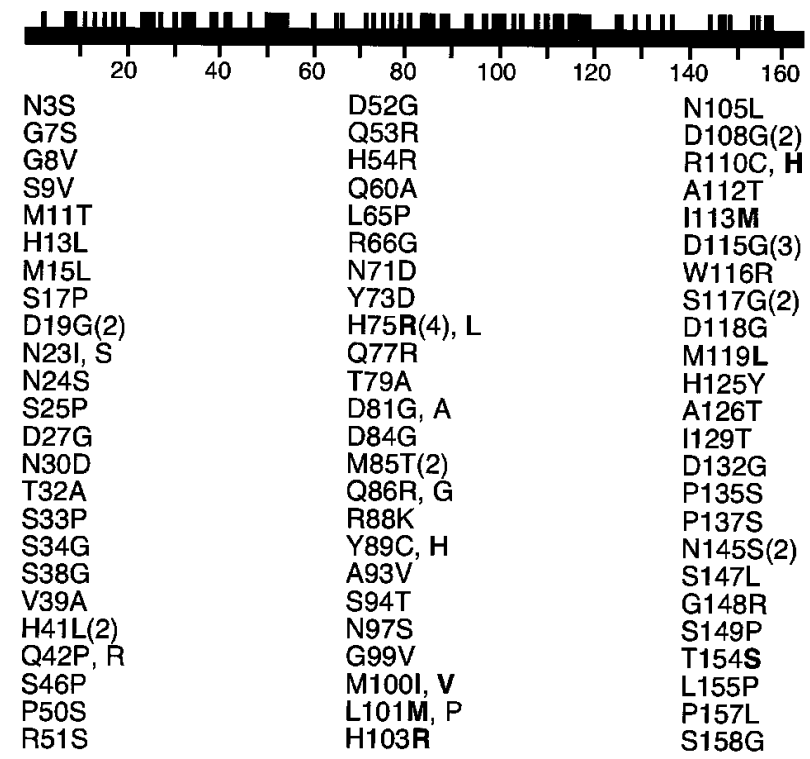

Fig. 2. Positions of residues in AvrPto altered by mutagenic polymerase chain reaction. Horizontal bar represents the 164 amino acid AvrPto protein. Each of the 72 vertical bars corresponds to the position of an altered residue. Substitutions are listed as wild-type residue, followed by its position and substituted residue. Numbers in parenthesis represent the number of times that particular substitution was identified. Letters in bold represent conservative changes. All others are considered nonconservative changes. 
Pto in the yeast two-hybrid system and in elicitation of the HR in plants expressing Pto.

\section{Pathogenicity assay of $P$. syringae pv. tabaci carrying mutant alleles of avrPto.}

To correlate elicitation of the HR with disease resistance, mutants were tested for their effects on the pathogenicity of $P$. syringae pv. tabaci on $N$. benthamiana expressing CaMV35S::Pto. The mutant alleles of avrPto were expressed from the wild-type avrPto promoter, mobilized into $P$. syringae pv. tabaci (the causal agent of wild-fire disease of tobacco), and inoculated into $N$. benthamiana with or without CaMV35S::Pto at two inoculum concentrations. Differences between $A$. tumefaciens- and $P$. syringae-mediated expression would be indicative of AvrPto mutants that were compromised in delivery into plant cells by $P$. syringae. Because transient expression of the mutant alleles of avrPto were conducted in $N$. benthamiana, $P$. syringae pv. tabaci was used instead of $P$. syringae pv. tomato in order to assay delivery of avrPto into the same host genotype.

Leaves of N. benthamiana plants expressing CaMV35S::Pto showed differences in response to $P$. syringae pv. tabaci, depending upon the avrPto allele and the inoculation density (Fig. 3B and Table 1). In contrast, disease occurred on wildtype $N$. benthamiana in response to $P$. syringae pv. tabaci, regardless of inoculation density or the mutant allele of avrPto it carried. Disease symptoms were absent following inoculation with $1 \times 10^{6} \mathrm{CFU} / \mathrm{ml}$ of $P$. syringae $\mathrm{pv}$. tabaci carrying wild-type avrPto or 11 of the 12 mutant alleles of avrPto that encode products that interacted with Pto in the yeast twohybrid system. The only exception was the mutant $a v r P t o_{\mathrm{H} 54 \mathrm{P}}$ (see below). At inoculum concentrations of $1 \times 10^{8} \mathrm{CFU} / \mathrm{ml}$ of $P$. syringae pv. tabaci carrying wild-type avrPto or the same 11 of the 12 mutant alleles elicited a HR in less than $16 \mathrm{~h}$ (Chang 1999). Infiltration of $1 \times 10^{8} \mathrm{CFU} / \mathrm{ml}$ of $P$. syringae pv. tabaci carrying avrPto $_{\mathrm{Y} 89 \mathrm{C}}$ resulted only in a weak HR in N. benthamiana expressing CaMV35S::Pto (Chang 1999).

$N$. benthamiana expressing CaMV35S::Pto showed disease symptoms 5 days postinoculation, following inoculation with $1 \times 10^{6} \mathrm{CFU} / \mathrm{ml}$ of $P$. syringae pv. tabaci carrying each of the nine mutant alleles of avrPto that encoded products that did not interact with Pto in the yeast two-hybrid system (Fig. 3B and Table 1). Inoculations with $1 \times 10^{8} \mathrm{CFU} / \mathrm{ml}$ of $P$. syringae pv. tabaci carrying each of these same nine avrPto mutants did not elicit a HR in $N$. benthamiana expressing CaMV35S::Pto (Chang 1999). Some of these mutants may not be delivered into the host cell, but this aspect of AvrPto activity could not be distinguished from the failure to elicit Ptomediated resistance because both result in no response by the plant. Therefore, there was a strong correlation between the effect of these mutants on the pathogenicity of $P$. syringae pv. tabaci and the ability of AvrPto mutants to interact with Pto in the yeast two-hybrid system.

The one exception to this correlation was the mutant allele

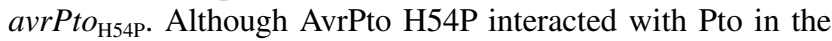
yeast two-hybrid system and elicited a HR following $A$. tumefaciens-mediated transient expression, disease did occur following inoculation with $1 \times 10^{6} \mathrm{CFU} / \mathrm{ml}$ of $P$. syringae $\mathrm{pv}$.

Table 1. Characteristics of amino acid substitution mutants of AvrPto

\begin{tabular}{|c|c|c|c|c|c|c|}
\hline Gene & $\begin{array}{l}\text { Source of } \\
\text { mutation }^{\text {a }}\end{array}$ & $\begin{array}{c}\text { Yeast two } \\
\text { interaction with } \text { Pto }^{\text {b }}\end{array}$ & $\begin{array}{l}\text { Agrobacterium spp.- } \\
\text { mediated expression }\end{array}$ & 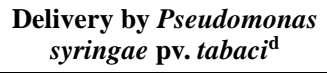 & $\begin{array}{l}\text { AvrPto } \\
\text { protein }^{\mathrm{e}}\end{array}$ & $\begin{array}{c}\text { Predicted } \\
\text { structural changes }\end{array}$ \\
\hline avrPto & None & + & HR & I & Yes & N/A \\
\hline Vector & None & - & $\mathrm{O}$ & $\mathrm{C}$ & N/A & N/A \\
\hline$H 54 P$ & PVX & + & HR & $\mathrm{C}$ & Yes & Yes \\
\hline$A 62 S$ & PVX & + & HR & I & Yes & Yes \\
\hline$W 116 T$ & PVX & + & HR & I & Yes & No \\
\hline$D 19 G$ & PCR & + & HR & I & Yes & No \\
\hline$S 25 P$ & PCR & + & HR & I & Yes & No \\
\hline$H 41 L$ & PCR & + & HR & I & Yes & No \\
\hline P50S & PCR & + & $\mathrm{HR}$ & I & Yes & No \\
\hline$Y 89 C$ & PCR & \pm & HR & I & Yes & No \\
\hline M100I & PCR & + & HR & I & Yes & No \\
\hline$D 115 G$ & PCR & + & HR & I & Yes & Yes \\
\hline D115G/A126T & PCR & + & HR & I & Yes & Yes \\
\hline$P 135 S$ & PCR & + & HR & I & Yes & No \\
\hline$L 43 P$ & PVX & - & $\mathrm{O}$ & $\mathrm{C}$ & Yes & Yes \\
\hline$Q 86 P$ & PVX & - & $\mathrm{O}$ & $\mathrm{C}$ & Yes & Yes \\
\hline$\widetilde{G} 8 \mathrm{~V} / Q 42 P$ & PCR & - & $\mathrm{O}$ & $\mathrm{C}$ & Yes & Yes \\
\hline$N 23 S / S 42 P$ & PCR & - & $\mathrm{O}$ & $\mathrm{C}$ & Yes & Yes \\
\hline$D 27 G / G 99 \mathrm{~V}$ & PCR & - & $\mathrm{O}$ & $\mathrm{C}$ & Yes & Yes \\
\hline$D 52 G / L 65 P$ & PCR & - & $\mathrm{O}$ & $\mathrm{C}$ & No & Yes \\
\hline N71D/W116R & PCR & - & $\mathrm{O}$ & $\mathrm{C}$ & Yes & Yes \\
\hline$Q 86 R$ & PCR & - & $\mathrm{O}$ & $\mathrm{C}$ & Yes & Yes \\
\hline Q86R/N145S & PCR & - & $\mathrm{O}$ & $\mathrm{C}$ & Yes & Yes \\
\hline
\end{tabular}

${ }^{\text {a }} \mathrm{PVX}=$ Potato virus $\mathrm{X} ; \mathrm{PCR}=$ polymerase chain reaction

${ }^{\mathrm{b}}+$ or $-=$ the interaction or lack of interaction, respectively, between the AvrPto mutant and Pto in the yeast two-hybrid system.

${ }^{\mathrm{c}} \mathrm{HR}=$ ability of the mutant to elicit a hypersensitive response (macroscopic plant cell death) when expressed via Agrobacterium spp.-mediated transient expression in N. benthamiana expressing CaMV35S::Pto; O = lack of a HR. Photographs of each of these interactions are available in Chang (1999).

${ }^{\mathrm{d}} \mathrm{I}$ or $\mathrm{C}=$ incompatibility (no disease symptoms) or compatibility (water-soaking lesions and discoloration of leaves), respectively, between Nicotiana benthamiana expressing CaMV35S::Pto and P. syringae pv. tabaci carrying each mutant.

e Detection of AvrPto protein in $P$. syringae pv. tabaci grown in Hrp-inducing media with anti-AvrPto antibodies. N/A = not applicable.

${ }^{\mathrm{f}}$ Protein three-dimensional structural changes predicted by Peptool 1.0 (Biotools, Edmonton, AB, Canada). 
tabaci carrying avrPto $_{\mathrm{H} 54 \mathrm{P}}$ (Fig. 3B), and infiltration of $1 \times 10^{8}$ $\mathrm{CFU} / \mathrm{ml}$ of $P$. syringae pv. tabaci carrying avrPto $_{\mathrm{H} 54 \mathrm{P}}$ did not result in a HR in $N$. benthamiana expressing CaMV35S::Pto (Chang 1999). To confirm this discrepancy, avrPto ${ }_{\mathrm{H} 54 \mathrm{P}}$ was mobilized into a normally virulent strain of the tomato pathogen $P$. syringae pv. tomato $\mathrm{T}_{1}$. The Pto-expressing cultivar Rio Grande 76R and the susceptible near-isogenic line $76 \mathrm{~S}$ were inoculated with $1 \times 10^{6} \mathrm{CFU} / \mathrm{ml}$ of $P$. syringae $\mathrm{pv}$. tomato strain $\mathrm{T}_{1}$ carrying wild-type avrPto, avrPto $\mathrm{H}_{\mathrm{H} 4 \mathrm{P}}$, or an empty pDSK519 vector. As expected, bacterial speck disease was observed on leaves of Rio Grande $76 \mathrm{~S}$ following separate inoculations of all three of the above $T_{1}$ strains. On Rio Grande 76R, P. syringae pv. tomato $\mathrm{T}_{1}$ expressing avrPto elicited a resistance response and no disease symptoms were observed. In contrast, inoculation of $P$. syringae pv. tomato $\mathrm{T}_{1}$ carrying $a v r P t o_{\mathrm{H} 54 \mathrm{P}}$ or pDSK519 resulted in disease of Rio Grande 76R (Fig. 3C). Therefore, Pto was unable to mediate a disease resistance response to $P$. syringae pv. tabaci or pv. tomato carrying avrPto $_{\mathrm{H} 54 \mathrm{P}}$ because AvrPto $\mathrm{H} 54 \mathrm{P}$ is either not delivered into the plant cells or its protein was not expressed or unstable in the cytoplasm of Pseudomonas spp.

\section{Expression of AvrPto mutant proteins in Hrp-inducing conditions.}

To determine the presence of mutant proteins of AvrPto, Western analysis was used to analyze expression of avrPto $_{\mathrm{H} 54 \mathrm{P}}$ and each of the other 20 avrPto mutant alleles in P. syringae pv. tabaci. Strains of $P$. syringae pv. tabaci carrying the mutant alleles were grown individually under Hrp-inducing and noninducing conditions (Huynh et al. 1989; Lindgren 1997). Protein was isolated from equal densities of cells, and AvrPto was detected with polyclonal rabbit anti-AvrPto antibodies. Samples were loaded equally. as determined by Coomassie staining.

Each mutant protein was expressed specifically in Hrpinducing conditions, except for AvrPto D52G/L65P, for which no protein was detected (Fig. 4A to C). Therefore, AvrPto H54P was expressed within the bacterial cell, suggesting it may not be delivered into the plant cell by $P$. $s y$ ringae. In contrast, AvrPto D52G/L65P was not recognized by Pto in the yeast two-hybrid system or in planta after $A$. tumefaciens-mediated transient expression and does not appear to be stable in Pseudomonas spp. The other $19 \mathrm{mu}-$ tant AvrPto proteins were expressed and stable. Therefore, the lack of resistance responses to $P$. syringae pv. tabaci carrying most of the mutant alleles of avrPto, which encoded products that did not bind Pto in the yeast two-hybrid system and did not elicit a HR by A. tumefaciens-mediated transient expression, is a result of the lack of recognition by Pto.

The differences in expression levels of mutant proteins of AvrPto in P. syringae pv. tabaci (Fig. 4) probably did not influence the differences in the ability to elicit the defense response. The yeast two-hybrid interactions with Pto were correlated with activities in planta for nearly all AvrPto mutants independent of expression levels. Also, levels of expressed AvrPto protein by the pathogen in minimal media may not reflect amounts translocated into the plant cell. The levels of translocated protein required for eliciting the defense response are not known. In Yersinia spp. strains capable of translocating YopE, only 2 to $3 \%$ of wild-type levels still show YopE cytotoxic effects, albeit delayed slightly (Frithz-Lindsten et al. 1995).

\section{DISCUSSION}

We mutagenized avrPto in order to identify regions within AvrPto for Pto-binding, elicit Pto-mediated resistance, and attain delivery by $P$. syringae. Deletion analysis identified a minimal region of 95 amino acids between the first 30 aminoterminal amino acids and the last 40 carboxy-terminal amino acids of AvrPto, which was sufficient for recognition by Pto in the yeast two-hybrid system. Sixty-five mutants of AvrPto with amino acid substitutions were tested for interactions with Pto in the yeast two-hybrid system, and 21 were characterized further in planta. There was an absolute correlation between binding in the yeast two-hybrid system and elicitation of a HR in planta by A. tumefaciens-mediated transient expression for all the tested mutants of avrPto. There also was a strong correlation between these two activities and the effect on pathogenicity of $P$. syringae pv. tabaci. Additionally, avrPto ${ }_{\mathrm{Y} 89 \mathrm{C}}$ encodes a product with a weakened ability to interact with Pto in the yeast two-hybrid system and showed a correspondingly weakened ability to elicit a HR in response to A. tumefaciensor $P$. syringae pv. tabaci-mediated expression. Therefore, the yeast two-hybrid interaction between AvrPto and Pto is representative of their interaction in planta.

AvrPto can tolerate many nonconservative amino acid substitutions and still interact with Pto. A total of 74 of the 164 residues of AvrPto were altered. Almost all were predicted to cause nonconservative amino acid changes. Yet, the majority of mutant proteins still interacted with Pto in the yeast twohybrid system. Eight different proteins with a total of 13 amino acid substitutions had lost the ability to interact with Pto. Six of these 13 residues, $\mathrm{G}^{8}, \mathrm{~N}^{23}, \mathrm{~S}^{27}, \mathrm{Q}^{42}, \mathrm{~W}^{116}$, and $\mathrm{N}^{145}$, also were altered in other mutant AvrPto proteins that interacted with Pto in the yeast two-hybrid system and/or were located within the first 30 amino acids that were dispensable for interaction with Pto. Consequently, only $\mathrm{L}^{43}, \mathrm{~S}^{46}, \mathrm{D}^{52}, \mathrm{~L}^{65}$, $\mathrm{N}^{71}, \mathrm{Q}^{86}$, and $\mathrm{G}^{99}$ seem to be important for interacting with Pto. Therefore, the interaction between Pto and AvrPto is not dependent upon a large proportion of the primary amino acid sequence of AvrPto.

Critical residues may be recognized directly by Pto or required for maintaining the structural integrity of AvrPto. Peptool 1.0 (Biotools, Edmonton, AB, Canada) was used to predict the secondary structure of AvrPto and the effects each substitution might have on AvrPto. All noninteracting mutant proteins of AvrPto had predicted changes relative to the predicted wild-type structure, suggesting that critical residues were required to maintain the structural integrity of AvrPto. Four noninteracting mutants were proline substitutions. Proline has a lower probability of residing in $\alpha$-helices than other residues because of its bulky, planar side chain. Q42P and L43P are predicted to cause breaks in the second predicted $\alpha$-helix, suggesting that this structural alteration may have influenced the interaction between AvrPto and Pto. $Q^{86}$, however, had three separate substitutions of proline, glycine, and arginine, all of which resulted in AvrPto mutants that could not interact with Pto. This may suggest that glutamine, at this specific position, in addition to its influence on the overall structure of AvrPto, is critical for interactions with Pto. 
Of the 12 AvrPto mutants that interacted with Pto in the yeast two-hybrid system, only four were predicted to have structural changes. The substitutions, H54P or A62S, were predicted to shorten a putative $\alpha$-helix only by one residue within the core of AvrPto sufficient for interactions with Pto. Substitution D115G in two AvrPto mutants disrupted an $\alpha$ helix near the breakpoint that defines the minimal region of AvrPto sufficient for Pto recognition.

AvrPto H54P did not elicit a HR in plants with a functional Pto gene when expressed by $P$. syringae pv. tabaci and pv. tomato. Western analysis confirmed that AvrPto H54P was expressed in the cytoplasm of $P$. syringae pv. tabaci when cells were grown in conditions shown to induce avirulence protein expression. Yet, when delivered in planta by A. tumefaciens, AvrPto H54P elicited a HR in N. benthamiana expressing CaMV35S::Pto and interacted with Pto in the yeast two-hybrid system. Therefore, a substitution of H54P does not interfere with the interaction between AvrPto and Pto in planta or ex planta. The different mechanisms for delivering AvrPto H54P into the host cell, T-DNA transfer versus protein translocation via the type III secretion system used by A. tumefa-

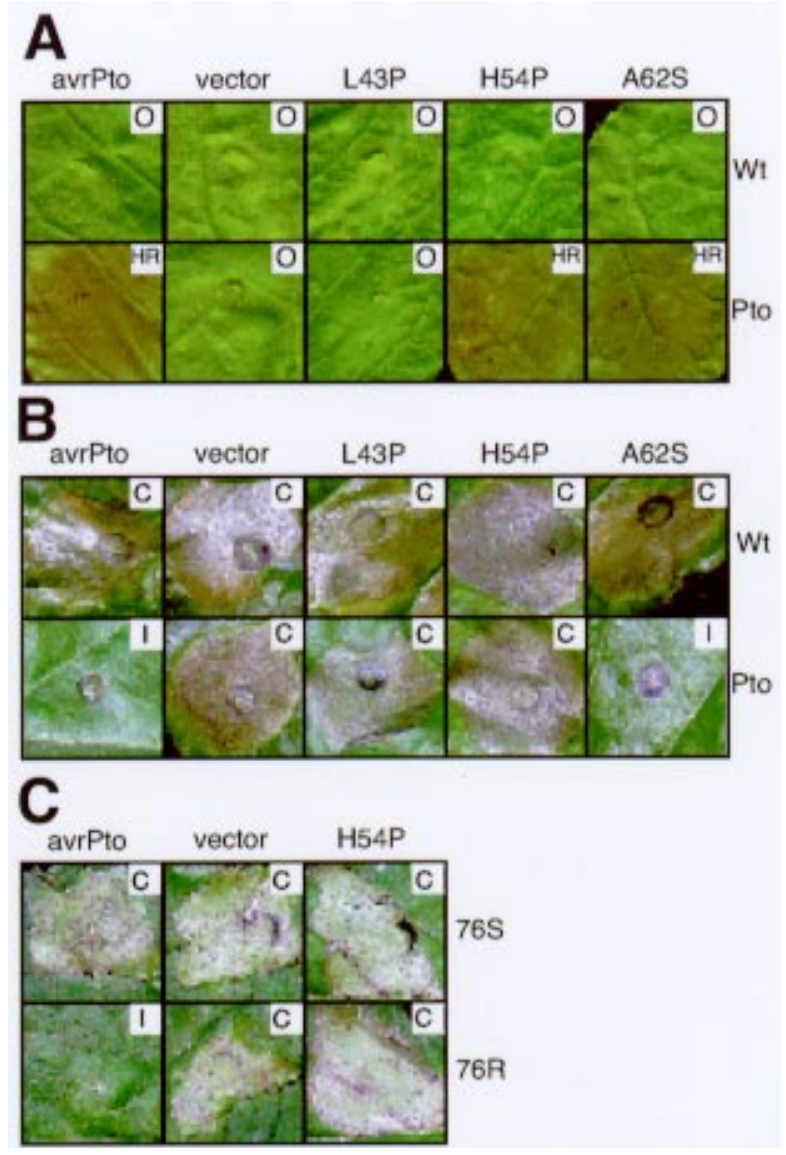

Fig. 3. Expression of representative mutants of AvrPto in planta. Leaves of Nicotiana benthamiana (Wt) and $N$. benthamiana expressing CaMV35S::Pto (Pto) were infiltrated with A, Agrobacterium tumefaciens or B, $1 \times 10^{6}$ Pseudomonas syringae pv. tabaci. $\mathbf{C}$, Leaves of tomato cultivars Rio Grande 76R and 76S were infiltrated with 1 x $10^{6} \mathrm{CFU} / \mathrm{ml}$ of $P$. syringae pv. tomato carrying avrPto $_{\mathrm{H} 54 \mathrm{P}} . \mathrm{HR}=$ hypersensitive response; $\mathrm{O}=$ no hypersensitive response; $\mathrm{C}=$ compatibility; $\mathrm{I}=$ incompatibility. Photographs were taken $\mathbf{A}$ and B, 5 days post inoculation and C, $24 \mathrm{~h}$ postinoculation. Results are representative of at least three separate infiltrations. ciens and Pseudomonas spp., respectively, may explain the disparity in their ability to elicit a HR in N. benthamiana expressing CaMV35S::Pto. Determinants required for delivering reporter proteins into host cells by the type III secretion pathway have been identified within secreted virulence factors of the mammalian bacterial pathogen Yersinia spp. The first 15 amino acids of YopE and the first 17 of YopH that fused to Cya were required to secrete the reporter protein out of the pathogen, and the fusion of an additional 50 and 71 residues, respectively, was required for translocation into macrophages (Sory et al. 1995). Either secretion or translocation of AvrPto could have been affected by the substitution H54P.

AvrPto D52G/L65P was not expressed or was rapidly degraded in the pathogen. The former is less likely because all avrPto mutant alleles were cloned as transcriptional fusions to the same avrPto promoter. It also is possible that the amino acid substitutions in AvrPto D52G/L65P led to a structure no longer recognized by these anti-AvrPto antibodies. This also is unlikely because these polyclonal antibodies were made with the entire AvrPto protein and reacted against a diverse set of functional and nonfunctional mutants of AvrPto. Nevertheless, we cannot exclude this possibility. Molecular chaperones of Shigella and Yersinia spp. promote stability of the type III secretion-dependent Ipa and Yop proteins, respectively, (Menard et al. 1994; Wattiau et al. 1994). IpaB and IpaC independently bind $\mathrm{IpgC}$ in the bacterial cytoplasm. Strains with mutations in IpgC show significantly lower levels of IpaB and IpaC, suggesting that IpgC promotes their protein stability (Menard et al. 1994). Likewise, DspB of the plant pathogen Erwinia amylovora has been suggested to be a chaperone of the Hrp-dependent pathogenicity factor DspA (Gaudriault et al. 1997). If AvrPto has a similar dependence on a chaperone for stability, it is possible that mutations at positions 52 and/or 65 prevent this interaction.

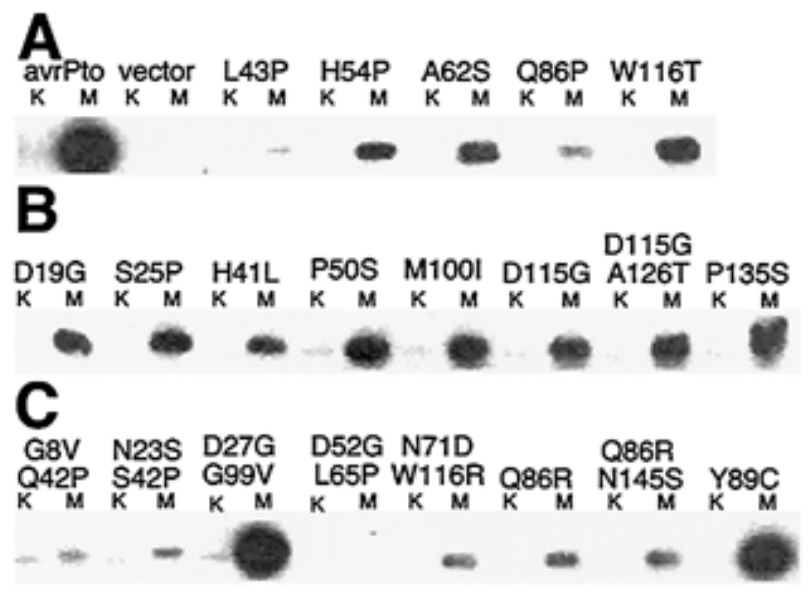

Fig. 4. Western analysis of Pseudomonas syringae pv. tabaci expressing mutant proteins of AvrPto. Cells were grown in King's B media (K) or minimal media (M). Proteins were detected with polyclonal rabbit antiAvrPto antibodies. The slightly smaller protein band visible in some King's B-grown cells represents a cross-reacting protein expressed in $P$. syringae pv. tabaci. A, Expression of AvrPto mutants identified from PVX::avrPto-infected plants exhibiting wild-type PVX symptoms. B, Expression of substitution mutants of AvrPto generated by mutagenic polymerase chain reaction-encoding products that interacted with Pto in the yeast two-hybrid system. C, Expression of substitution mutants of AvrPto that interacted weakly or did not interact with Pto in the yeast two-hybrid system. 
Biological selection on PVX::avrPto was used as an effective method to identify mutations in avrPto with altered activities. cDNAs of avrPto had mutations when isolated from plants exhibiting wild-type PVX symptoms (Tobias et al. 1999; this study). This strategy, however, has a low efficiency; only 5 to $10 \%$ of infected plants showed altered phenotypes. A much lower percentage were infected with PVX carrying avrPto with missense mutations. In retrospect, our analyses of the mutant alleles of avrPto provides an explanation for this observation. Very few residues of AvrPto appear to affect its recognition by Pto, thus reducing the probability of altering important residues. Mutagenic PCR is the converse of the PVX::avrPto selection system in that it is efficient in creating point mutations in avrPto, yet few had altered biological activity. Sixty clones were isolated, and the number of altered residues accounted for almost $44 \%$ of the entire protein. Alterations were distributed evenly throughout, and none were deletions, insertions, or nonsense mutations. Only $13.3 \%$ of the mutant AvrPto proteins, however, were affected in Pto binding. The yeast two-hybrid system provides a facile and relevant method for analyzing the interaction between AvrPto and Pto. A reverse yeast two-hybrid system has become available and could be used for selecting noninteracting proteins (Vidal et al. 1996). This system is appropriate for more extensive analyses of AvrPto residues necessary for Pto-recognition.

Subsequent to the conclusion of our studies, mutational analysis of AvrPto also has been reported by Shan et al. (2000). Their findings mostly parallel our own, in that Shan et al. identified nine mutants of AvrPto with single amino acid substitutions that failed to interact with Pto in the yeast twohybrid assay and did not reduce the growth of $P$. syringae pv. tomato in resistant cultivars of tomato. In both studies, three amino acids (at positions 43, 86, and 99) were shown to be critical for recognition of AvrPto by Pto. Shan et al. identified six additional single amino acid substitutions (at positions 11, $46,72,94,96$, and 105) that also appeared to be important for recognition. Our study indicated the presence of 10 more residues that were probably critical; however, we were unable to definitively locate these because seven of the nine mutants of AvrPto that failed to interact with Pto carried multiple amino acid substitutions. None of these mutants of AvrPto had substitutions common to those identified by Shan et al. Our data contrast with those of Shan et al. at three positions (11, 94, and 105). We also created mutants with substitutions at these positions; however, we did not observe an effect on the interaction with Pto. For positions 94 and 105, these inconsistencies could be a result of the differences in the residues introduced. Shan et al. created S94P and N105K mutants, whereas we created S94T and N105L substitutions. Our substitutions were considered nonconservative but were likely to be less disruptive than the substitutions characterized by Shan et al. The M11T substitution was identified in both studies. The reason for the inconsistency between the two experiments is unclear, although in our studies M11T occurred in a triple mutant (M11T, T79A, and R88K). Therefore, considering the data from both studies, only 19 residues of this 164 amino acid peptide have so far been implicated as important for the interaction with Pto. In contrast, our study demonstrated that substitutions in at least 60 amino acid positions did not disrupt the interaction between AvrPto and Pto in the yeast twohybrid system or in planta.
These mutant alleles also will be valuable for analyzing virulence functions conferred by AvrPto. Most, if not all, avirulence genes are expected to confer some advantage to the pathogen under certain conditions. AvrBs 2 of Xanthomonas campestris pv. vesicatoria is required for maximal growth in susceptible pepper hosts (Kearney and Staskawicz 1990). AvrE and avrRpml are required for maximal growth by specific strains of $P$. syringae pv. tomato and $P$. syringae pv. maculicolia, respectively, on susceptible hosts (Lorang et al. 1994; Ritter and Dangl 1995). Likewise, virulence activities have been attributed to some avirulence genes of Xanthomonas oryzae pv. oryzae and to AvrRpt2 of P. syringae in strain DC3000 (Bai et al. 2000; Chen et al. 2000). Recently, a small AvrPto-dependent increase in growth of $P$. syringae pv. tomato in planta has been observed (Chang et al. 2000; Shan et al. 2000). Shan et al. (2000) characterized their nine mutants for virulence activity as measured by growth in susceptible plants. The three mutants with substitutions clustered in close proximity to each other between residues 94 and 99 were not affected in virulence, whereas the remaining six were affected.

\section{MATERIALS AND METHODS}

\section{Cloning, mutagenesis and yeast two-hybrid analysis of avrPto.}

PCR was used to amplify the ORF of avrPto from pACT2::avrPto (Scofield et al. 1996) with Pfu polymerase (Stratagene, La Jolla, CA, U.S.A.) and the primer sequences 5'-CCATGGGAAATATATGTGTCGGCGG and 5'-CTGGAG TCATTGCCAGTTACGGTACGG that anneal to nucleotide positions 1 and 495 of avrPto, respectively. PCR conditions were $30 \mathrm{~s}$ at $92^{\circ} \mathrm{C}, 30 \mathrm{~s}$ at $55^{\circ} \mathrm{C}$, and $30 \mathrm{~s}$ at $70^{\circ} \mathrm{C}$. The amplification product was cloned into the vector pCR Blunt (Invitrogen, Carlsbad, CA, U.S.A.).

Deletions were introduced systematically by amplifying the ORF of avrPto with Pfu polymerase and primers complimentary to the avrPto DNA sequence (GenBank accession no. L20425.1) at positions every $30 \mathrm{bp}$. Primers for the $5^{\prime}$ end started at positions $1,31,61,91,121,148$, and 181 of the ORF. Primers for the $3^{\prime}$ end started at 495, 402, 372, and 342 of the ORF. Primers for the $5^{\prime}$ and $3^{\prime}$ ends included NcoI and XhoI sites, respectively. Products were recloned into pCR Blunt, sequenced to verify the fidelity of the sequence, and then cloned into pACT2 (Clontech, Palo Alto, CA, U.S.A.) as NcoI-XhoI fragments.

Mutagenic PCR was performed on the ORF of avrPto cloned in pCR Blunt by amplification for 30 cycles of $30 \mathrm{~s}$ at $92^{\circ} \mathrm{C}, 30 \mathrm{~s}$ at $57^{\circ} \mathrm{C}$, and $30 \mathrm{~s}$ at $72^{\circ} \mathrm{C}$ in mutagenic buffer $(50.0$ $\mathrm{mM} \mathrm{KCl}, 10.0 \mathrm{mM}$ Tris-HCl [pH 9.0], 0.1\% Triton X-100, 2.0 $\mathrm{mM} \mathrm{MgCl}_{2}, 0.5 \mathrm{mM} \mathrm{MnCl}_{2}, 0.2 \mathrm{mM}$ dATP, $0.2 \mathrm{mM}$ dGTP, $1.0 \mathrm{mM}$ dCTP, $1.0 \mathrm{mM}$ dTTP, and five units Taq polymerase) with the M13F and M13R primer sequences 5'-CACGACGT TGTAAAACGAC and 5'-GGATAACAATTTCACACAGG. The mutagenic PCR products were digested with NcoI and XhoI and cloned into compatible sites of pACT2. Individual clones were amplified by PCR with primers that annealed to regions of pACT2 flanking the multiple cloning site 5'CAGGGATGTTTAATACCACTACAATG and 5'-GCACAGT TGAAGTGAACTTGCGGG. PCR products were sequenced with the KlenTaq-1 cycle sequencing kit (Rematech, Davis, 
CA, U.S.A.) on a Licor 4200L automated sequencer. Mutations were confirmed by multiple sequencing of both strands.

Yeast two-hybrid analysis was conducted with the Matchmaker GAL4 system, following the protocols of the manufacturer (Clontech). Alleles of avrPto were cotransformed individually with Pto cloned in pAS2.1 (Scofield et al. 1996) into yeast strain Y190. Cotransformed yeast cells were selected by growth on selective media and replicated on Whatman No. 1 filters (Maidstone, Kent, U.K.) prior to assaying for activation of $\beta$-galactosidase with the substrate 5-bromo-4chloro-3-indolyl $\beta$-D-galactoside (X-gal). Positive and negative interactions were confirmed multiple times with independently transformed yeast cells.

\section{A. tumefaciens-mediated transient expression of mutant alleles of avrPto.}

Clones of pACT2 carrying mutant alleles of avrPto were digested with $\mathrm{NcoI}$, filled in with Klenow, according to the manufacturer (New England Biolabs, Beverly, MA, U.S.A.), digested with XhoI, and ligated to pMD1 (a pBI121-based binary vector in which the GUS gene had been replaced with a multiple cloning site) that had been digested with a SmaI and XhoI. Clones were transformed into A. tumefaciens strain A281 by the freeze-thaw method. Overnight cultures were diluted 1:10 in Luria-Bertani medium plus $200 \mu \mathrm{M}$ acetosyringone plus $20 \mu \mathrm{g}$ of kanamycin per $\mathrm{ml}$ and grown at $28^{\circ} \mathrm{C}$ overnight with shaking. Cultures were washed in infiltration media $(1 / 10 \times$ MS salts, $1 / 10 \times \mathrm{B} 5$ vitamins, $20 \mathrm{mM}$ morpholineethanesulfonic acid, $2 \%$ [wt/vol] sucrose, $1 \%$ [wt/vol] glucose, and $200 \mu \mathrm{M}$ acetosyringone at $\mathrm{pH}$ 5.4) and diluted to an optical density (OD) at $600 \mathrm{~nm}$ of 1.0. A. tumefaciens was pressure infiltrated into leaves of approximately 6-week old $N$. benthamiana plants. Inoculated plants were grown at $21^{\circ} \mathrm{C}$ for 4 to 5 days and assessed for development of the HR by visual inspection.

\section{Assays of $P$. syringae pathogenicity.}

Clones of pACT2 carrying mutant alleles of avrPto were digested with XhoI, filled in with Klenow, digested with NcoI, and ligated into pBlueE3 (Salmeron and Staskawicz 1993) that had been digested with $N c o$ I and SmaI, which fused the ORFs of the avrPto alleles to the native avrPto promoter. These clones were digested further with $K p n I$ (or the isoschizomers Acc65I or Asp718) and XbaI and ligated to the corresponding sites in the broad-host range vector pDSK519. Clones were mobilized into $P$. syringae pv. tabaci (causal agent of wild-fire disease on tobacco) or $P$. syringae pv. tomato by triparental mating with the helper plasmid pRK2013. Transformed cells were grown overnight in King's B media (20 g of Difco protease peptone No. 3 [Detroit, MI, U.S.A.] per liter, $1.5 \mathrm{~g}$ of $\mathrm{K}_{2} \mathrm{HPO}_{4}$ per liter, $1.6 \mathrm{~g}$ of $\mathrm{MgSO}_{4} \times 7 \mathrm{H}_{2} 0$ per liter, and $10 \mathrm{ml}$ of glycerol [pH 7.2] per liter) containing $50 \mu \mathrm{g}$ of rifampicin per $\mathrm{ml}$ and $20 \mu \mathrm{g}$ of kanamycin per $\mathrm{ml}$ at $28^{\circ} \mathrm{C}$ with shaking. Cells were washed in $10 \mathrm{mM} \mathrm{MgCl}_{2}$ and resuspended in $10 \mathrm{mM} \mathrm{MgCl}_{2}$ at $\mathrm{OD}_{600}$ of 1.0. P. syringae pv. tabaci were diluted to either $1 \times 10^{8} \mathrm{CFU} / \mathrm{ml}$ or $1 \times 10^{6}$ $\mathrm{CFU} / \mathrm{ml}$. P. syringae pv. tomato was diluted to $1 \times 10^{6}$ $\mathrm{CFU} / \mathrm{ml}$. Cell suspensions were infiltrated into leaves of approximately 6-week old $N$. benthamiana or 4-week old tomato plants. Plants were grown at $21^{\circ} \mathrm{C}$ and inspected after 1 day for development of HR or 4 to 5 days for development of disease symptoms.

\section{AvrPto induction in Hrp-inducing conditions.}

Expression of mutant proteins of AvrPto in P. syringae pv. tabaci was induced in media that simulate the environment of the leaf apoplast (Huynh et al. 1989). Equal densities of cells expressing mutant proteins of AvrPto, as determined by $\mathrm{OD}_{600}$, were resuspended individually in $1 \times$ sodium dodecyl sulfate (SDS)-loading buffer and lysed by boiling. Protein was separated by SDS-polyacrylamide gel electrophoresis (PAGE) on a $12 \%$ acrylamide gel, transferred to Immobilon-P (Millipore, Burlington, MA, U.S.A.) in $10 \%$ ethyl alcohol and $10 \mathrm{mM}$ CAPS (3-[cyclohexylamino]-1-propano sulfonic acid), $\mathrm{pH} 11$, and probed with rabbit polyclonal anti-AvrPto antibodies (B. Gabel and R. Michelmore, unpublished). Bound antibodies were detected with peroxidase-conjugated goat anti-rabbit antibodies and visualized with the ECL detection kit (Amersham Pharmacia Biotech, Arlington Heights, IL, U.S.A.).

\section{Assays of potato virus $X$ infection.}

PVX::avrPto (Tobias et al. 1999) was digested with SpeI and phenol extracted twice and chloroform extracted once. RNA was synthesized with the mMESSAGE mMACHINE kit, following the directions of the manufacturer (Ambion, Austin, TX, U.S.A.). RNA was pipetted onto cotyledons of 1to 2-week-old tomato or 3- to 4-week-old N. benthamiana plants and rubbed gently with small glass vials. Phenotypes were assessed 2 weeks after infection. Total RNA was extracted from one infected leaf (Ausubel et al. 1987). Reverse transcription-PCR and cloning were performed as described previously (Tobias et al. 1999).

\section{ACKNOWLEDGMENTS}

We thank K. Lambert for his assistance on the secondary-structure prediction of mutant proteins of avrPto; J. Gardner and J. Luke for sequencing support; G. Pearson and J. Alvine for caring of plants; B. Gabel for antiAvrPto antibodies; and J. Rathjen, C. Wong, and A. Bernal for critical reading of the manuscript. The research was supported by NSF Cooperative Agreement BIR-8920216 to the Center for Engineering Plants for Resistance Against Pathogens (CEPRAP) and by CEPRAP corporate associate Sandoz Seeds.

\section{LITERATURE CITED}

Ausubel, F. M., Brent, R., Kingston, R. E., Moore, D. D., Seidman, J. G., Smith, J. G., and Struhl, K. 1987. Current Protocols in Molecular Biology. Greene and Wiley Interscience, New York.

Bai, J., Choi, S.-H., Ponciano, G., Leung, H., and Leach, J. E. 2000. Xanthomonas oryzae pv. oryzae avirulence genes contribute differently and specifically to pathogen aggressiveness. Mol. Plant-Microbe Interact. 13:1322-1329.

Chang, J. H. 1999. Molecular characterization of the interactions between tomato and Pseudomonas syringae pv. tomato. Ph.D. thesis. University of California-Davis, U.S.A.

Chang, J. H., Rathjen, J. P., Bernal, A. J., Staskawicz, B. J., and Michelmore, R. W. 2000. avrPto enhances growth and necrosis caused by Pseudomonas syringae pv. tomato in tomato lines lacking either Pto or Prf. Mol. Plant-Microbe Interact. 13:568-571.

Chen, Z., Kloeck, A. P., Boch, J., Katagiri, F., and Kunkel, B. N. 2000. The Pseudomonas syringae avrRpt2 gene product promotes pathogen virulence from inside the plant cells. Mol. Plant-Microbe Interact. 13:1312-1321.

Dangl, J. L., Dietrich, R. A., and Richberg, M. H. 1996. Death don't have no mercy: Cell death programs in plant-microbe interactions. Plant Cell 8:1793-1807.

Frederick, R. D., Thilmony, R. L., Sessa, G., and Martin, G. B. 1998. 
Recognition specificity for the bacterial avirulence protein AvrPto is determined by Thr-204 in the activation loop of the tomato Pto kinase. Mol. Cell 2:241-245.

Frithz-Lindsten, E., Rosqvist, R., Johansson, L., and Forsberg, A. 1995. The chaperone-like protein YerA of Yersinia pseudotuberculosis stabilizes YopE in the cytoplasm but is dispensable for targeting to the secretion loci. Mol. Microbiol. 16:635-647.

Fromant, M., Blanquet, S., and Plateau, P. 1995. Direct random mutagenesis of gene-sized DNA fragments using polymerase chain reaction. Anal. Biochem. 224:347-353.

Gaudriault, S., Malandrin, L., Paulin, J. P., and Barny, M. A. 1997. DspA, an essential pathogenicity factor of Erwinia amylovora showing homology with AvrE of Pseudomonas syringae, is secreted via the Hrp secretion pathway in a DspB-dependent way. Mol. Microbiol. 26:1057-1069.

Gopalan, S., Bauer, D. W., Alfano, J. R., Loniello, A. O., He, S. Y., and Collmer, A. 1996. Expression of the Pseudomonas syringae avirulence protein AvrB in plant cells alleviates its dependence on the hypersensitive response and pathogenicity (Hrp) secretion system in eliciting genotype-specific hypersensitive cell death. Plant Cell 8:1095-1105.

Ham, J. H., Bauer, D. W., Fouts, D. E., and Collmer, A. 1998. A cloned Erwinia chrysanthemi Hrp (type III protein secretion) system functions in Escherichia coli to deliver Pseudomonas syringae Avr signals to plant cells and to secrete Avr proteins in culture. Proc. Natl. Acad. Sci. USA. 95:10206-10211.

Hardt, W. D., and Galan, J. E. 1997. A secreted Salmonella protein with homology to an avirulence determinant of plant pathogenic bacteria. Proc. Natl. Acad. Sci. USA 94:9887-9892.

Herbers, K., Conrads-Strauch, J., and Bonas, U. 1992. Race-specificity of plant resistance to bacterial spot disease determined by repetitive motifs in a bacterial avirulence protein. Nature 356:172-174.

Hueck, C. J. 1998. Type III protein secretion systems in bacterial pathogens of animals and plants. Microbiol. Mol. Biol. Rev. 62:379-433.

Huynh, T. V., Dahlbeck, D., and Staskawicz, B. J. 1989. Bacterial blight of soybean: Regulation of a pathogen gene determining host cultivar specificity. Science 245:1374-1377.

Innes, R. W., Bent, A. F., Kunkel, B. N., Bisgrove, S. R., and Staskawicz, B. J. 1993. Molecular analysis of avirulence gene avrRpt 2 and identification of a putative regulatory sequence common to all known Pseudomonas syringae avirulence genes. J. Bacteriol. 175:4859-4869.

Kearney, B., and Staskawicz, B. J. 1990. Widespread distribution and fitness contribution of Xanthomonas campestris avirulence gene avrBs2. Nature 346:385-386.

Keen, N. T., Tamaki, S., Kobayashi, D., Gerhold, D., Stayton, M., Shen, H., Gold, S., Lorang, J., Thordal-Christenson, H., Dahlbeck, D., and Staskawicz, B. 1990. Bacteria expressing avirulence gene D produce a specific elicitor of the soybean hypersensitive reaction. Mol. PlantMicrobe Interact. 3:112-121.

Leister, R. T., Ausubel, F. M., and Katagiri, F. 1996. Molecular recognition of pathogen attack occurs inside of plant cells in plant disease resistance specified by the Arabidopsis genes RPS2 and RPM1. Proc. Natl. Acad. Sci. USA 93:15497-15502.

Leung, D. W., Chen, E., and Goeddel, D. V. 1989. A method for random mutagenesis of a defined DNA segment using a modified polymerase chain reaction. Techniques 1:11-15.

Lindgren, P. B. 1997. The role of hrp genes during plant-bacterial interactions. Annu. Rev. Phytopathol. 35:129-152.

Lorang, J. M., Shen, H., Kobayashi, D., Cooksey, D., and Keen, N. T. 1994. avrA and avrE in Pseudomonas syringae pv. tomato PT23 play a role in virulence on tomato plants. Mol. Plant-Microbe Interact. $7: 508-515$
Martin, G. B., Brommonschenkel, S. H., Chunwongse, J., Frary, A., Ganal, M. W., Spivey, R., Wu, T., Earle, E. D., and Tanksley, S. D. 1993. Map-based cloning of a protein kinase gene conferring disease resistance in tomato. Science 262:1432-1436.

Menard, R., Sansonetti, P., Parsot, C., and Vasselon, T. 1994. Extracellular association and cytoplasmic partitioning of the IpaB and IpaC invasins of S. flexneri. Cell 79:515-525.

Mudgett, M. B., and Staskawicz, B. J. 1999. Characterization of the Pseudomonas syringae pv. tomato AvrRpt2 protein: Demonstration of secretion and processing during bacterial pathogenesis. Mol. Microbiol. 32:927-941.

Rathjen, J. P., Chang, J. H., Staskawicz, B. J., and Michelmore, R. W. 1999. Constitutively active Pto induces a Prf-dependent hypersensitive response in the absence of avrPto. EMBO J. 18:3232-3240.

Ritter, C., and Dangl, J. L. 1995. The avrRpml gene of Pseudomonas syringae pv. maculicola is required for virulence on Arabidopsis. Mol. Plant-Microbe Interact. 8:444-453.

Rommens, C. M. T., Salmeron, J. M., Oldroyd, G. E. D., and Staskawicz, B. J. 1995. Intergeneric transfer and functional expression of the tomato disease resistance gene Pto. Plant Cell 7:1537-1544.

Ronald, P. C., Salmeron, J. M., Carland, F. M., and Staskawicz, B. J. 1992. The cloned avirulence gene avrPto induces disease resistance in tomato cultivars containing the Pto resistance gene. J. Bacteriol. 174:1604-1611.

Salmeron, J. M., and Staskawicz, B. J. 1993. Molecular characterization and hrp dependence of the avirulence gene avrPto from Pseudomonas syringae pv. tomato. Mol. Gen. Genet. 239:6-16.

Scofield, S. R., Tobias, C. M., Rathjen, J. P., Chang, J. H., Lavelle, D. T., Michelmore, R. W., and Staskawicz, B. J. 1996. Molecular basis of gene-for-gene specificity in bacterial speck disease of tomato. Science 274:2063-2065.

Shan, L., He, P., Zhou, J.-M., and Tang, X. 2000. A cluster of mutations disrupt the avirulence but not the virulence functions of AvrPto. Mol. Plant-Microbe Interact. 13:592-598.

Sory, M. P., Boland, A., Lambermont, I., and Cornelis, G. R. 1995. Identification of the YopE and YopH domains required for secretion and internalization into the cytosol of macrophages, using the cyaA gene fusion approach. Proc. Natl. Acad. Sci. USA 92:11998-12002.

Swords, K. M., Dahlbeck, D., Kearney, B., Roy, M., and Staskawicz, B. J. 1996. Spontaneous and induced mutations in a single open reading frame alter both virulence and avirulence in Xanthomonas campestris pv. vesicatoria avrBs2. J. Bacteriol. 178:4661-4669.

Tang, X. Y., Frederick, R. D., Zhou, J. M., Halterman, D. A., Jia, Y. L., and Martin, G. B. 1996. Initiation of plant disease resistance by physical interaction of AvrPto and Pto kinase. Science 274:20602063.

Tobias, C. M., Oldroyd, G. E. D., Chang, J. H., and Staskawicz, B. J. 1999. Plants expressing the Pto disease resistance gene confer resistance to recombinant PVX containing the avirulence gene AvrPto. Plant J. 17:41-50.

Van den Ackerveken, G., Marois, E., and Bonas, U. 1996. Recognition of the bacterial avirulence protein AvrBs3 occurs inside the host plant cell. Cell 87:1307-1316.

Vidal, M., Brachmann, R. K., Fattaey, A., Harlow, E., and Boeke, J. D. 1996. Reverse two-hybrid and one-hybrid systems to detect dissociation of protein-protein and DNA-protein interactions. Proc. Natl. Acad. Sci. USA 93:10315-10320.

Wattiau, P., Bernier, B., Deslee, P., Michiels, T., and Cornelis, G. R. 1994. Individual chaperones required for Yop secretion by Yersinia. Proc. Natl. Acad. Sci. USA 91:10493-10497.

Yang, Y., and Gabriel, D. W. 1995. Xanthomonas avirulence/pathogenicity gene family encodes functional plant nuclear targeting signals. Mol. Plant-Microbe Interact. 8:627-631. 